\title{
Osteodystrophy in chronic liver diseases
}

\author{
Pasquale Mansueto $\cdot$ Antonio Carroccio • \\ Aurelio Seidita · Gaetana Di Fede · Antonio Craxì
}

Received: 20 September 2011/ Accepted: 4 January 2012/Published online: 13 January 2012

(C) SIMI 2012

\begin{abstract}
Osteoporosis and osteomalacia are, to date, among the most common metabolic diseases in the world. Lately, an association between metabolic bone diseases and chronic liver disease has been increasingly reported, inducing many authors to create a new nosographic entity known as 'hepatic osteodystrophy.' The importance of such a condition is further increased by the morbidity of these two diseases, which greatly reduce the quality of life because of frequent fractures, especially vertebral and femoral neck ones. For this reason, early identification of high-risk patients should be routinely performed by measuring bone mass density. The explanation for the association between bone diseases and chronic liver disease is still uncertain, and involves many factors: from hypogonadism to use of corticosteroid drugs, from genetic factors to interferon therapy. To date, few studies have been
\end{abstract}

P. Mansueto · A. Seidita

Dipartimento di Medicina Interna e Specialistica,

Università di Palermo, Palermo, Italy

P. Mansueto $(\square)$

Dipartimento di Medicina Clinica e Delle Patologie Emergenti, Azienda Ospedaliera Universitaria Policlinico 'P. Giaccone',

Via del Vespro, 141, 90127 Palermo, Italy

e-mail: pasquale.mansueto@unipa.it

\section{A. Carroccio}

Unità Operativa di Medicina Interna, Ospedali Riuniti

di Sciacca e Università di Palermo, Palermo, Italy

G. Di Fede

Dipartimento di Discipline Chirurgiche ed Oncologiche, Università di Palermo, Palermo, Italy

A. Craxì

Dipartimento Biomedico di Medicina Interna e Specialistica, Università di Palermo, Palermo, Italy conducted, and all with a small number of patients to establish definitive conclusions about the possible treatment, but some evidence is beginning to emerge about the safety and efficacy of bisphosphonates.

Keywords Osteoporosis - Osteomalacia ·

Chronic liver disease $\cdot$ Bisphosphonates

\section{Introduction}

Metabolic bone diseases, i.e., osteomalacia and osteoporosis, are frequent complications of patients affected with chronic liver disease (CLD), to the point that some authors have coined the term 'hepatic osteodystrophy' to mean the complex of structural and metabolic changes of bone in patients with both cirrhotic and non-cirrhotic CLD. Among these, osteoporosis is particularly important because more frequent than osteomalacia, and often responsible for fractures, which, although most often asymptomatic, contribute, in any case, to morbidity and reduction of the quality of life. Unlike vertebral fractures, femoral neck and extremities ones, symptomatic by definition, are less frequent in these patients, because, even in subjects not suffering from CLD, they occur about 10 years after the former, that is, beyond the life expectancy of many patients with CLD [1].

\section{Osteomalacy and chronic liver diseases}

Osteomalacia is a metabolic osteopathy characterized by a reduced amount of bone mineral content due to lack of mineralization. This osteopathy, which potentially can be found in several pathological conditions, is usually caused 
by reduced availability or abnormal metabolism of vitamin D. Osteomalacia, although in the past reported in more than $60 \%$ of patients with primary biliary cirrhosis (PBC), is currently demonstrated in only a small percentage of patients with CLD, especially in advanced cholestatic ones, with severe intestinal malabsorption, and in geographical areas with limited sunlight exposure. The changes in prevalence of this disease, occurring in recent decades, reflect, probably, the use of different diagnostic criteria, errors in selection of patients included in trials, and nutritional improvements. However, the gold standard for the diagnosis of osteomalacia is still considered to be bone biopsy, because not all patients with low serum levels of vitamin D suffer from this metabolic disease [2].

As a matter of fact, vitamin $\mathrm{D}$ includes a group of fatsoluble prohormones (Fig. 1). The group includes two compounds with very similar biological activity: cholecalciferol (D3), derived from cholesterol and synthesized in animals, and ergocalciferol (D2), of vegetable origin. In humans, vitamin D3 is produced by ultraviolet irradiation of the skin from a precursor, 7-dehydrocholesterol, which is in the subcorneal layer. Adequate exposure to sunlight grants endogenous production of cholecalciferol able to satisfy the needs of the whole organism; hence, dietary supplement is not necessary. The digestive tract absorption of vitamin D from food sources, animal (D3) or vegetable (D2), follows similar processes employed by other fat-soluble vitamins. Vitamin D is incorporated into the micelles, formed by interaction between hydrolyzed lipids and bile, cross the intestinal epithelium, and finally is incorporated in chylomicrons, which, in turn, enter the lymphatic circulation. In various tissues, especially in the liver, hydroxylation of cholecalciferol produce 25-hydroxycholecalciferol (25-OHD), which then passes into the general circulation, and interacts with a specific transport protein (vitamin $\mathrm{D}$ binding protein, DBP), synthesized by the liver. In the kidney, the 25-OHD may undergo two different hydroxylation reactions, caused by different hydroxylase, 1-hydroxylase and 24-hydroxylase, generating, respectively, 1,25-dihydroxycholecalciferol (1,25-OHD), the active form of vitamin D, and 24,25-dihydroxycholecalciferol (24,25-OHD), the inactive form. As the liver is involved in the production of bile, vitamin D3 absorption (fat-soluble vitamin) and blood transport, through the DBP synthesis, and 25-hydroxylation, which produces $25-\mathrm{OHD}$, it would be easy to assume a high prevalence of osteomalacia in patients suffering from CLD, not confirmed, as mentioned above, in more recent years. In fact, adults obtain most of their vitamin D3 from cutaneous photoconversion of 7-dehydrocholesterol, and patients with cholestasis seem to have normal skin photoconversion processes. In addition, intestinal vitamin D absorption is altered only in the presence of a severe cholestasis. The succeeding 25-hepatic vitamin D3 hydroxylation has so far been little studied in human, but in experimental animals, with severe cholestasis, does not seem impaired. In any case, about $2 / 3$ of patients with liver cirrhosis and $96 \%$ of terminal cirrhotic patients waiting for liver transplant have low serum levels of vitamin D3, even if without any demonstrated osteomalacia. However, vitamin D derivatives may be involved in cell proliferation, differentiation, and immunomodulation. Vitamin D inhibits some types of matrix

Fig. 1 Vitamin D metabolism

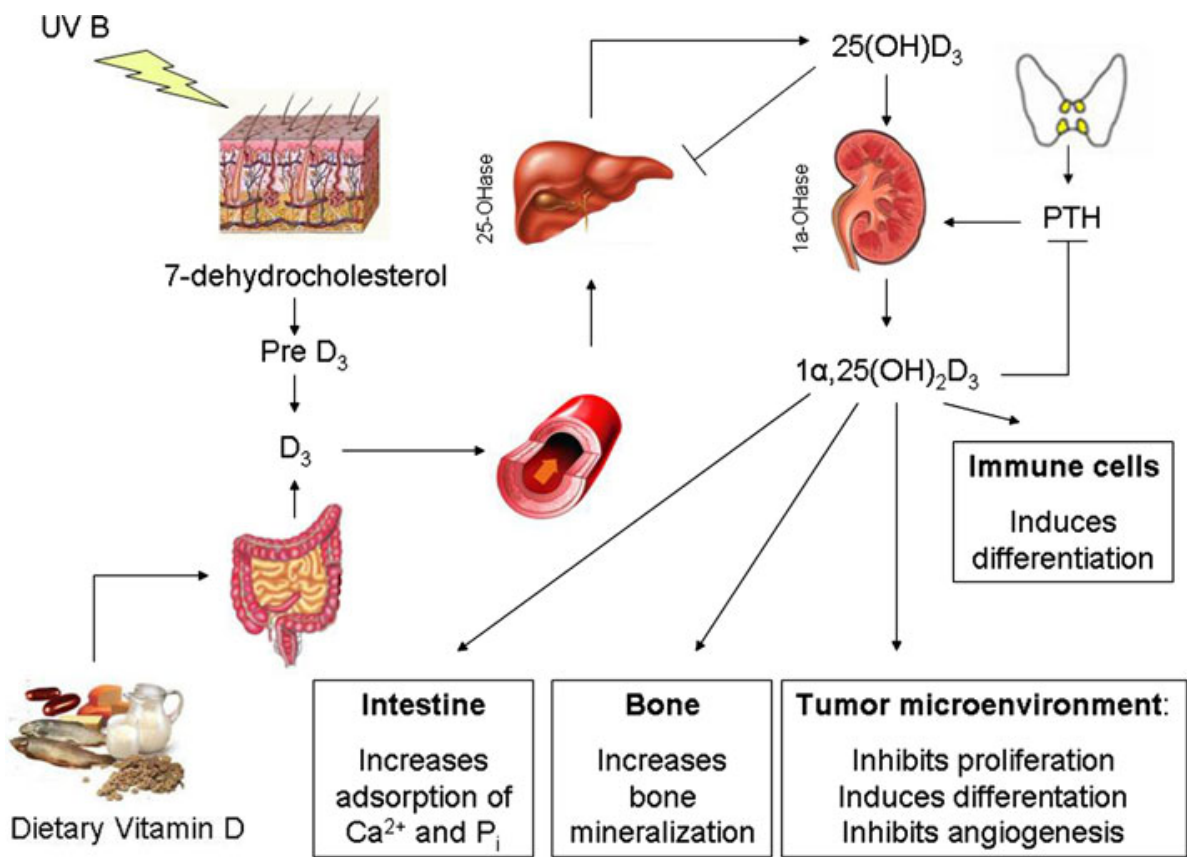


metalloproteinases (MMP, a family of zinc-dependent endoproteinases that are involved in the degradation of extracellular matrix components) and induces their inhibitors. Finally, suppression of fibroblast proliferation and collagen production are other vitamin $\mathrm{D}$ effects. These data are relevant to CLD, and vitamin D deficiency can lead to progression of hepatic fibrosis. Therefore, in view of the increasingly recognized beneficial effects of adequate levels of vitamin D, measurement of 25-OHD levels and its replacement may be considered as a part of the overall management of patients with CLD [3].

\section{Osteoporosis and chronic liver diseases}

Osteoporosis is defined as a value of bone mineral density (BMD), measured by dual-energy X-ray absorptiometry (DEXA), $<2.5$ standard deviations below the average of a young adult population control bone mass peak (corresponding to the normal bone mass peak) ( $T$ score $<-2.5)$, whereas osteopenia is defined as $T$ score values between -1 and -2.5 . 'Normal' is a BMD with a $T$ score better than -1 . Although the risk of fractures increases gradually with decreasing BMD, many other clinical risk factors are important, all independent from BMD, such as advanced age, menopause, body mass index (BMI) $<19 \mathrm{~kg} / \mathrm{m}^{2}$ (expression of malnutrition or poor nutrition), alcohol intake $>3 \mathrm{U} /$ day $(1 \mathrm{U}=8 \mathrm{~g}$ alcohol), smoking, oral corticosteroid therapy $>5 \mathrm{mg}$ /day of prednisone for at least 3 months, previous fragility fractures, and maternal history of hip fracture. The most important of these is surely represented by previous fragility fractures. Once a vertebral fracture occurs, the risk of further vertebral fracture increases by 10 times, while risk of a subsequent hip fracture increases by 2.3 times [4].

Osteoporosis occurs in patients with CLD, affects mainly trabecular bone, and has been characterized by low bone turnover, with reduced osteoblast function, and low serum osteocalcin levels. However, there is a certain heterogeneity regarding the prevalence of osteoporosis in CLD patients, which depends on patient selection, liver disease etiology and stage, and additional risk factors for osteoporosis. Actually, most studies have been focused on bone disease in chronic cholestatic liver disease, mainly in patients with $\mathrm{PBC}$, and in bone disease before and after liver transplantation. In contrast, few studies evaluate bone disorders in other CLD, such as hereditary hemochromatosis, viral hepatitis, and alcoholic liver disease. In addition, many osteoporosis additional risk factors, including hypogonadism, malnutrition, excessive alcohol intake and use of corticosteroid drugs (i.e., treatment of autoimmune liver diseases, as $\mathrm{PBC}$ and primary sclerosing cholangitis (PSC),) may be found in patients affected with CLD.
Moreover, many patients with $\mathrm{PBC}$ are, by definition, postmenopausal women, sometimes also affected with inflammatory bowel diseases, which significantly increase osteoporosis risk. It is still questionable whether liver cirrhosis and cholestasis are, themselves, independent risk factors for osteoporosis, since this topic has been not yet completely studied [5].

\section{Pathophysiology of osteoporosis in chronic liver diseases}

The amount of bone mass depends on the balance between two opposite processes: bone resorption, originated by osteoclasts, and bone formation, induced by osteoblasts. As a consequence, if resorption exceeds formation, there is a negative balance, which results in bone loss and osteoporosis. The mechanisms resulting in osteoporosis pathogenesis in liver disease have not been completely elucidated. Some studies indicate an increased bone resorption, although most point towards a decreased bone formation. Indeed, impaired osteoblast function, resulting in lower mean wall thickness and a defect in matrix synthesis, as well as a low bone formation rate, that affects, mainly, trabecular one, have been reported. These data are consistent with the decreased serum levels of osteocalcin, a biochemical marker of bone formation [6].

Probably, the main role in the pathophysiology of osteoporosis in these conditions is played by leading pathogenic factors, such as gender and hormone changes, i.e., hypogonadism, which is frequent in patients with hereditary hemochromatosis, cirrhosis and alcoholic liver disease. However, biological mechanisms underlying osteoporosis of patients with CLD are complex, and nowadays only slightly understood, and include, perhaps, also genetic and acquired factors.

Hypogonadism seems to play a particularly important role among osteodystrophy pathogenic mechanisms in CLD. Low-free testosterone levels as well as levels of estrogens that have a relative increase point to hypogonadism in men with liver cirrhosis. Alteration of sensitivity of the hypothalamic-pituitary-gonadal axis seems to be, at least in part, responsible for the reduction of free testosterone levels, depending on the lower gonadal production. As a matter of fact, patients affected with liver cirrhosis show FSH and LH levels only slightly increased, but certainly not so high as expected because of a severe lack of circulating sex hormones. As testosterone is metabolized to estrogen, a long duration of hypogonadism in men is often associated with a reduction in circulating estrogens, and consequent reduced bone turnover, with decreased osteosynthesis [7]. Since these endocrine alterations described above play a heavy clinical role in male, it is clear that 
most studies on this aspect have been conducted in cirrhotic males. However, limited data, obtained in female patients with cirrhosis, show that levels of testosterone, FSH and LH levels are significantly reduced. The link between CLD and osteoporosis is emphasized by the hormonal changes that can lead to secondary amenorrhea in young women affected with liver cirrhosis [8]. Among genetic factors, it must be consider collagen type $I \alpha 1$, vitamin D receptor (VDR), estrogen receptor and low density lipoprotein receptor-binding protein 5 (LRP5) genes, but their polymorphism has not yet been associated with an increased, specific fracture risk, even in patients without CLD [9].

As mentioned above, a possible acquired dissociation between bone formation and resorption may explain bone loss, subsequent to bone mass peak overtaking. Increase in osteoclastic activity, resulting in enhanced bone resorption, is mediated by osteoclastogenic pro-inflammatory cytokines, such as IL-1 and TNF, often involved both in hepatic inflammation and fibrosis. Lately, receptor activator of NF$\kappa \mathrm{B}$ (RANK) protein and RANK ligand (RANKL), in addition to osteoprotegerin (OPG), have been involved in osteoclastic bone resorption mechanisms in patients with no hepatic osteoporosis, while assessment of their involvement in CLD patients has, so far, produced contradictory results [10].

Contrariwise, reduction of bone formation, observed in patients with CLD, is associated with low serum levels of IGF-1, a protein, primarily synthesized in the liver, known to be involved in bone remodeling and maintenance of bone mass, as a trophic and proliferative factor for osteoblasts. A clear association between IGF-1 serum levels and osteoporosis, in CLD in humans, has not been, yet clearly demonstrated [11].

Vitamin $\mathrm{K}$ is also involved in bone metabolism, by carboxylation of glutamic acid residues of bone proteins, such as osteocalcin, but there are no literature data about vitamin $\mathrm{K}$ deficiency role in osteoporosis pathogenesis in hepatic diseases [12].

Although indirect (unconjugated) bilirubin inhibits the activity of osteoblasts and their function, both in vitro and in vivo studies, conducted in animal models and patients with CLD, show no correlation between total, direct and indirect bilirubin levels and BMD [13]. Recent experiments demonstrate that lithocholic acid (LCA), a monohydroxylated secondary bile acid, synthesized mainly from the intestinal bacterial 7-dehydroxylation of chenodeoxycholic acid, has deleterious effects on human osteoblasts, both in relation to their viability and regarding the potential damaging effects of LCA on vitamin D pathways, through ligation to VDR. Actually, significant decreased osteoblast viability is demonstrated when adding LCA to osteoblasts culture media. This detrimental effect of LCA on osteoblasts viability is decreased or completely abolished by adding proteins, i.e., human albumin, to the culture media. These results can be explained by the binding capacity of albumin, which may have, partially or completely, absorbed or sequestered free LCA, and therefore, decreases its detrimental effect on cell viability. Therefore, this observation is in accordance with the well-known capacity of albumin as a bile acid transporter, and it could be proposed that the amount of circulating albumin is one of the critical factors to explain the harmful effects on bone of circulating bile acids retained in patients with chronic cholestasis, data which are in agreement with the higher prevalence of osteoporosis and bone fractures observed in patients with $\mathrm{PBC}$ and severe cholestasis. As a matter of fact, a lower serum albumin concentration may be detected in osteoporotic patients affected with PBC with osteoporosis than in not osteoporotic ones. In addition, LCA interaction with VDR modifies expression of VDR-mediated genes, such as vitamin D 24-hydroxylase (CYP24A), bone gamma-carboxyglutamate protein $(B G L A P)$, and receptor activator for $\mathrm{NF} \kappa \mathrm{B}$ ligand (RANKL, TNFSF11), used as indicators of vitamin D catabolism, osteoblast maturation, and regulation of osteoclast differentiation and activation, respectively [14].

\section{Prevalence of osteoporosis and fractures in chronic liver diseases}

There is some heterogeneity regarding prevalence of osteoporosis and fractures in patients affected with CLD, which partly depends on patient selection and diagnostic criteria. Approximately $30 \%$ of patients with liver disease have osteoporosis, with a higher prevalence in patients affected with $\mathrm{PBC}$, the latter having two additional risk factors: chronic cholestasis and female gender. The prevalence of fractures in patients with liver disease ranges from 7 to $35 \%$. Fractures are more prevalent in postmenopausal women than in young women and men and in patients with autoimmune hepatitis treated with corticosteroid drugs [1].

Biliary diseases: primary biliary cirrhosis and primary sclerosing cholangitis

An etiologic relationship between PBC and osteoporosis is still very controversial, because patients suffering from this disease are classically postmenopausal women, themselves, therefore, at high risk of osteoporosis, sometimes also affected with inflammatory bowel diseases, another condition that significantly increases osteoporosis risk. In patients with $\mathrm{PBC}$, osteoporosis has been recently reported to be up to $37 \%$ in a series of 185 Spanish female patients, the lumbar spine being more severely affected than the 
femoral neck. The prevalences of vertebral, non-vertebral, and overall fractures are 11.2, 12.2, 20.8\%, respectively. Non-vertebral fractures included mostly wrist fractures, followed by lower limb fractures; only one patient had a hip fracture. Vertebral fractures are associated with osteopenia and osteoporosis, with a $T$ score lower than -1.5 , whereas osteopenia and osteoporosis are associated with the severity of liver damage. The clear-cut correlation between vertebral fracture and a $T$ score lower than -1.5 , observed in these patients may indicate that this densitometric measurement is a useful criteria for prescribing agents to prevent further loss of bone mass and development of new fractures [15]. Similarly, Menon et al. [16] found that as many as $20 \%$ of PBC patients, in a large series from the Mayo Clinic, have osteoporosis. In both studies, osteoporosis is more frequent in PBC than in healthy control women. Mounach et al. evaluating a series of 33 women with PBC and 66 healthy control subjects, find the mean $T$ score to be lower in the PBC group as compared to healthy controls, with a significant statistical difference both for lumbar spine and total hip involvement. The prevalence of osteoporosis is $51.5 \%$ in the PBC group versus $22.7 \%$ in healthy controls, with a statistically significant difference. A positive relationship between BMD, values of BMI and 25-OHD has been highlighted, whereas a negative one has been reported for menopausal status, duration of disease, and parathyroid hormone (PTH) levels [17].

A recent UK study confirms a substantially increased osteoporosis and fracture risk in this particular group of patients when compared with age-matched healthy controls, with a prevalence of $43 \%$ for osteoporosis and $22 \%$ for fractures. There are approximately twofold relative increases in the risk of any fracture, hip fracture and ulna/ radius fracture for the $\mathrm{PBC}$ cohort compared with general population [18].

All these observations are of interest, because as other studies suggest, osteoporosis is not a specific complication of $\mathrm{PBC}$, its frequency being the one expected in a population of middle-aged postmenopausal women [19].

In contrast, nowadays, it seems that liver damage may play a relevant role, resulting in bone loss. Two recent studies propose a role for the severity and duration of cholestasis in the development of osteoporosis. Thus, a higher Mayo risk score, calculating the severity of PBC, and advanced histological stage of the liver disease, in addition to advanced age and lower BMI, are the independent risk factors for osteoporosis in a series of women with PBC [20]. Similarly, Menon et al. report that age, BMI, advanced stage of the disease, and history of fractures are the only independent indicators of osteoporosis. Furthermore, the serum bilirubin level is the only independently associated variable with the rate of bone loss over time [16]. In these studies, the authors also demonstrate that agents, such as prednisone and budesonide, used for the treatment of the liver disease, may also affect bone mass, and that the risk for fracture, in $\mathrm{PBC}$, is associated mainly with low bone mass, and, indirectly, through this condition, with the severity and duration of liver disease. Unlike PBC, PSC occurs at a younger age, and affects mainly male subjects. Most patients with PSC also have inflammatory bowel disease, which significantly increases the risk of developing osteoporosis, particularly Crohn's disease. This risk is even greater in patients with IBD treated with corticosteroids. The prevalence of osteoporosis in PSC stands at $20.3 \%$, with wide variability among the studies, while risk of fracture, approximately $15 \%$, correlates with increasing age, more advanced biliary disease and duration of a coexisting and associated inflammatory bowel disease [21].

Another even more recent evaluating a cohort of 237 patients with PSC, demonstrates that osteoporosis affects $15 \%$ of patients and occurs 23.8 -fold more frequently in those with PSC than expected from a matched population. Age (54 years or older), low BMI and inflammatory bowel disease duration positively correlates with the presence of osteoporosis. Osteoporosis can be detected in $75 \%$ of patients with all three risk factors, but in only $3.1 \%$ of those without all of them. Patients with PSC lose $1 \%$ of bone mass per year. This rate of bone loss is significantly associated with the duration of the inflammatory bowel disease. Therefore, the authors conclude that old age, low BMI and long duration of inflammatory bowel disease can be used to identify patients with PSC who might have the most benefit from measurements of bone density and treatments for bone diseases [22].

Finally, some recent data showing similar bone histomorphometric changes in PBC and PSC, suggest that both decreased bone formation and increased resorption contribute to bone disease in female patients with $\mathrm{PBC}$ and PSC, whereas decreased bone formation rather than increased resorption may be an important pathogenic mechanism more relevant in male patients. These histomorphometric studies were performed in patients with advanced PBC and PSC undergoing liver transplantation, and thus further studies are necessary to determine whether similar histomorphometric changes occur in patients with earlier stages of liver disease [23].

\section{Other chronic liver diseases}

In non-cholestatic liver disorders, like hereditary hemochromatosis and other more prevalent liver diseases, such as cirrhosis, viral chronic hepatitis and alcoholic liver disease, there is less information. 


\section{Hereditary hemochromatosis}

From available data, about $30 \%$ of patients with hereditary hemochromatosis have osteoporosis. In a recent study of 87 patients $(80 \%$ male) with hereditary hemochromatosis, a quarter of them have osteoporosis and $41 \%$ have osteopenia, even in subjects without cirrhosis and hypogonadism. In this disease, the deposits of iron deposits may be responsible for low bone formation, due to the direct lesion-producing effects of iron on osteoblast activity [24].

\section{Cirrhosis}

Several studies conducted over the past two decades demonstrate that the prevalence of osteoporosis in patients with liver cirrhosis, albeit with wide variability among the studies, is around $33 \%$, ranging from 20 to $100 \%$, especially in patients with end-stage liver disease before transplantation. These differences in prevalence reflect, probably, differences in age, etiology and severity of liver disease, nutritional status (expressed as BMI), and in the hypogonadism status of studied patients. Liver cirrhosis seems to increase the risk of fractures by about two times [25].

\section{Viral chronic hepatitis}

Regarding viral chronic hepatitis, and its treatment with ribavirin and interferon, there are conflicting results. A small study assessing bone mass in chronic viral hepatitis, mostly hepatitis $\mathrm{C}$ virus, have shown that non-cirrhotic hepatitis $\mathrm{C}$ virus infected patients do not differ from controls [26], whereas in another recent study, BMD was low in patients with chronic non-cirrhotic hepatopathy, suffering from chronic viral infection by hepatitis $\mathrm{C}$ virus. The study, however, has no control group, and there are no details about alcoholic intake [27]. Ribavirin and interferon therapy, used in the treatment of patients with HCV-related chronic active hepatitis, may have possible negative effects on BMD of these subjects, as initially shown in some studies [28]; but it is not confirmed with a demonstrated increase in BMD, shown in those patients who achieve a sustained serologic response [29].

\section{Alcoholic liver disease}

Bone disease in alcoholic liver disease has depicted more uniform results, since excess alcohol intake is a known independent risk factor for osteoporosis. Reduction in the grade of bone formation has been observed in alcoholic patients by bone biopsies, with low serum levels of osteocalcin during alcohol intake, which normalizes with abstinence [30]. Some authors demonstrate that alcoholic men, with or without mild liver disease, have significantly lower lumbar BMD than controls, and 29\% have osteoporosis. The study also reports the prevalence of radiological vertebral fractures to be up to $36 \%$. In this series, a previous history of non-vertebral fractures is high, ribs being a frequent location, since they occurred in $26 \%$ of patients. The authors suggest that traumatic fractures may account for a great proportion of fractures in the alcoholic population, although a fragility component cannot be ruled out. Most of the studies are focused on alcohol consumption without considering the contribution of liver disease to the fracture risk [31].

\section{Liver transplant}

Patients who undergo liver transplant show a post-transplant high prevalence of osteoporosis and fractures. Osteoporosis has become a major cause of co-morbidity in these patients, as their longtime survival is considerably increased in recent years. After liver transplantation, there seem to be two main phases in post-transplant bone disease: the early and the late post-transplantation periods. Before liver transplantation, there is a low bone turnover state, supported by histomorphometric analysis of bone biopsies and biochemical parameters. Shortly after liver transplantation, in the first 3 months, there is a significant and quantitatively large increase in bone turnover, substantiated by histomorphometric data, and an early increase in the biochemical markers of bone resorption that exceeds bone formation markers. Taken together, the earliest period of rapid bone loss and high rate of fractures is associated with a high bone turnover, with an uncoupling of resorption and formation. The normalization of liver function and a superimposed effect of immunosuppressive therapy, reliant on fairly high doses of glucocorticoids combined with cyclosporin A or tacrolimus, with their systemic effects, give rise to the picture during the early post-transplant period. The second and late phase, which generally appears 6 months after transplantation, is characterized by an increase in both histologic parameters and biochemical markers of bone formation. At this time, bone loss at the lumbar spine has stopped, and BMD has begun to increase spontaneously. Similarly, there is an increase in the PTH serum concentrations comparable to those of creatinine, as well as an increase in circulating osteocalcin. This secondary hyperparathyroidism, likely related to the decline of renal function, associated with cyclosporin A administration, may be a major contributor to the disorder of bone remodeling. These features occur while patients are maintained on cyclosporin A alone, or when combined with a low glucocorticoids dose. Therefore, factors involved in bone turnover in this second phase after transplantation are the normalization of liver function, and 
the gradual reduction in glucocorticoids, which are responsible for an increase in bone formation, as well as a secondary hyperparathyroidism, related to cyclosporin A administration [32].

In post-transplanted patients, there is a rapid bone loss within the first 6 months after transplantation, being as high as was pointed out to $6 \%$ in the lumbar spine at 3 months, and a frequency of fragility fractures, from minor trauma or apparently non-traumatic ones, both vertebral, symptomatic or not, and non-vertebral, femoral and/or of extremities, of about to $21 \%$, most of which within first 2 years after transplant, especially in women, female, elderly, cholestatic, and alcoholic patients, and particularly in those with osteoporosis and fragility fractures before transplantation. Most of post-transplant fractures, unlike pre-transplant ones, seem to be symptomatic, and, most commonly, vertebral, followed by ribs, with a minor prevalence of hip and extremities, probably because predominantly cortical bone sites are, in fact, less susceptible to osteoporotic fractures when compared with trabecular ones [33]. An etiology of this metabolic disorder is multifactorial, and risk factors contributing to it are both pre-transplant and post-transplant ones. Among pre-transplant risk factors, pre-transplant fragility fracture is more predictive for post-transplant recurrent fractures compared to the simple values of BMD. However, BMD measurement, of course, is also crucial in these patients, because low BMD values, before and after transplant, are associated with a higher frequency of fractures. In addition, there is widespread consensus on osteoporosis and fracture risk in post-transplant patients, as determined by age ( $>45$ years for women, $>65$ years for men), gender (female, although some studies showed an increased susceptibility to osteoporosis and fractures in males patients), menopausal status (postmenopausal), type of pre-transplant liver disease (increased risk for cholestatic or alcohol related disorders,) and exposure to corticosteroids in the pre-transplant phase. Other possible pre-transplant risk factors, for which there is no unanimous consensus, in themselves considered as risk factors for posttransplant osteoporosis and fractures, are 25-OHD and PTH serum levels, bone turnover markers, and biochemical parameters of liver (with reference also to the Child-Pugh class of membership, with class $\mathrm{C}$ at greater risk of osteoporosis than class A) and kidney function. The most important among post-transplant factors is represented by acute osteopenia that occurs immediately after transplant, with a significant decrease of BMD in first 3 months, and a return to pre-transplant values only after about 2 years [34].

An important contributing cause of rapid bone loss in the immediate postoperative period of these patients is probably the use of high doses of corticosteroids and other immunosuppressive agents, such as cyclosporin A and tacrolimus, immobility and poor nutrition. Cumulative steroid doses, administered in early post-transplant, and, in this delicate phase, at particularly high dosage, has been repeatedly implicated in pathogenesis of acute bone loss observed in these patients, while current use of lower steroids doses after transplant, as shorter time of hospitalization and immobilization, explain the sharp reduction of osteoporosis and fracture frequency in the post-transplant patient reported in several studies. Contrariwise, the effects of calcineurin inhibitors (i.e., tacrolimus), administered after liver transplant as anti-rejection drugs, on bone turnover, via increased PTH serum levels from a decrease in the glomerular filtration rate, are still under examination as it is difficult to separate possible bone loss effects of these drugs from those of corticosteroids used in anti-rejection treatment regimens. However, it seems that patients treated with tacrolimus have an earlier recovery of mineral content and trabecular bone structure as compared to patients taking cyclosporin A, but effects of both on bone loss in the early post-transplant period remain unclear. Both, however, seem to increase turnover and bone resorption, cyclosporin A, perhaps, more than tacrolimus [35].

To our knowledge, there are no studies assessing bone disease in liver transplant recipients under immunosuppressive agents, such as rapamycin, mycophenolatemofetil, and daclizumab. Finally, post-transplant variables that do not seem to have any effect on the prevalence of fractures after intervention are waiting time before the procedure, hospitalization length, immunosuppressive doses used, both daily and cumulative, and episodes of organ rejection [36].

\section{Measurement of BMD and diagnosis of osteoporosis}

The presence of risk factors for the development of osteoporosis should be evaluated in patients with CLD, including the following: advanced age, early menopause, secondary amenorrhea of more than 6 months, male hypogonadism, BMI lower than $19 \mathrm{~kg} / \mathrm{m}^{2}$, chronic alcohol intake, smoking, treatment with glucocorticoids $(5 \mathrm{mg} /$ day of prednisone or over for more than 3 months), previous fragility fractures, and family history of osteoporotic fracture. Despite some differences, most of guidelines and recommendations agree about DEXA determinations, as routine screening test, in all CLD patients, especially if major risk factors for osteoporosis can be evidenced, especially and in patients affected with chronic cholestasis and cirrhosis. Other possible indications to BMD measurement are hereditary hemochromatosis, and postmenopausal $\mathrm{HCV}+$ women, especially before IFN-based therapy, before and after hepatic transplant, and before steroids or cyclosporin A treatment. In all these cases, if an osteoporosis osteopenic framework is diagnosed, a medical treatment should be immediately started. BMD should be 
measured in patients with liver cirrhosis, both in biliary (i.e., PBC and PSC) and in non-biliary one, before and after hepatic transplant and before steroids or cyclosporin A treatment. Indications to BMD measurement are less clear in patients with cholestatic liver disease not waiting for transplant. For example, the guidelines of the American Gastroenterological Association suggest that BMD should be measured in all patients with $\mathrm{PBC}$ at diagnosis time, while others recommendations suggest BMD measurement only in cholestatic patients with a bilirubin greater than three times the upper limit of normal range. Unfortunately, there is still no agreement about the frequency of BMD measurements during the follow-up of patients. If no osteoporosis has been diagnosed, but in the presence of risk factors, there is probably no need to repeat BMD measurement DEXA more often than once every 2-3 years. Contrariwise, clinical conditions associated with a rapid bone loss, such as in cholestatic patients (i.e., PBC and PSC), with more than one risk factor for osteoporosis, and in those recently initiating high-dose corticosteroid therapies, the screening should be performed in a shorter time of approximately every year. This schedule is also recommended for cirrhotic patients with advanced clinical stage and those potentially eligible for liver transplantation. Considering then, that osteoporosis may also be the first clinical manifestation of an underlying cholestatic liver disease, it is advisable to screen for anti-mitochondrial antibodies in all osteoporotic patients with a low BMD and high cholestasis markers (gamma-glutamyltranspeptidase, $\gamma$-GT, and alkaline phosphatase) [37, 38]. Lateral X-rays of the dorsal and lumbar spine should also be carried out to reveal vertebral fractures. In addition, circulating serum levels of calcium, phosphorous, 25-hydroxyvitamin D 25-OHD and PTH serum levels should be assessed. Disturbances in thyroid and gonadal function should be ruled out in particular cases. Individual response to anti-osteoporotic treatment can be monitored assessing biochemical markers of bone turnover. However, there is not much information on changes in bone markers in therapeutic trials in patients with CLD, nor are there any practical guidelines for their use in the clinical management of postmenopausal osteoporosis. It seems they are not useful neither to stratify fracture risk nor to assess deterioration of bone health during follow-up. The indication of undercalcified transilial bone biopsy is advisable only if a mineralizing defect (i.e., osteomalacia) is suspected, which is, at present, a very rare condition in CLD [37, 38].

\section{Treatment of osteoporosis in chronic liver diseases}

In all CLD patients, especially those with severe cholestasis (bilirubin $>3^{\prime}$ for $>6$ months), the factors contributing to bone loss should, as far as possible, be reduced to a minimum, utilizing such basic preventive measures as stopping alcohol intake and smoking. In general, physical activity should be encouraged as much as possible, as specific exercises aimed to improve the BMD and the mechanics of the spine. However, the optimal exercise program for osteoporosis in patients with CLD or after liver transplantation has not yet been determined. Specific recommendations should be given in patients with increased risk of falling. In addition, as a baseline prevention, an adequate calcium/vitamin D intake (see below), by diet or pharmacological replacement, must be commenced by the patients. Current recommendations suggest starting osteoporosis treatment in all patients affected with CLD liver chronic disease, especially if aged $>65$ years, or if $7.5 \mathrm{mg}$ or more of steroid drugs are required daily for more than 3 months, although there is a possibility of an increased osteoporotic risk for lower steroid doses as well. In younger patients, suffering from CLD, treatment is indicated if BMD shows $T$ score values of -2.5 . Likewise, it seems reasonable to treat all patients as soon as possible after liver transplantation, and before transplantation if they have osteoporosis. Although serum and urine markers of bone turnover have been used in the assessment and treatment monitoring of patients affected with postmenopausal osteoporosis, there are few studies about their use in osteoporotic patients with CLD, and, actually, they should not be routinely obtained in all patients. The treatment of osteoporosis in patients with CLD is, essentially, based on the results of large trials conducted in women with postmenopausal osteoporosis. Contrariwise, there are, to date, only a few small studies specifically conducted in patients with CLD, particularly affected with $\mathrm{PBC}$; ones, that, moreover, consider as primary outcome, the BMD increase rather than frequency reduction of fragility fractures [39].

\section{Diet, calcium and vitamin D}

Whenever possible, a balanced diet should be prescribed, since patients with advanced liver disease frequently have little appetite and are malnourished. Supplements of calcium (1,000-1,500 mg/day) and vitamin D (260 $\mu \mathrm{g}$ of 25-hydroxyvitamin D every 2 weeks, or vitamin D3 with a dose of 400-800 IU/day or 5,000 IU/week, orally) should be provided. However, there are very few studies on the effectiveness of supplementation with calcium and vitamin $\mathrm{D}$, and as to their optimal dose and formulation in these patients with CLD. Nevertheless, vitamin D deficiency should be corrected to obtain a 25-OHD serum values of at least $25-30 \mathrm{ng} / \mathrm{mL}$. In patients with documented associated malabsorption should use higher vitamin $\mathrm{D}$ doses. The intestinal absorption of vitamin D may be reduced in patients receiving resins, such as cholestyramine. The dose 
of glucocorticoids should be adjusted to the minimum necessary [40].

\section{Bisphosphonates}

Bisphosphonates (alendronate, etidronate, ibandronate, and risidronate), drugs act via inhibition of bone resorption (Fig. 2), are all used in treatment of postmenopausal osteoporosis, most often in combination with calcium and vitamin D. Oral bisphosphonates are poorly absorbed in the intestine; they are not metabolized and rapidly cleared from the circulation. Approximately $50 \%$ of the absorbed fraction concentrates in the skeleton, and the remainder is excreted via the urine. There are, however, very few studies about their use in patients with cirrhosis, especially with PBC, so data about their safety and effectiveness are still inadequate. Furthermore, the physician must evaluate osteoporosis prevalence in patient-specific liver disease, the need for treatment in the light of natural history of disease in the single patient (life expectancy), and the potential hepatotoxicity of the treatment, both drug-related (consider hepatic and renal function) and unpredictable, together with gastric mucosal problems (i.e., portal hypertensive gastropathy) and varices. In patients with PBC, alendronate, more than etidronate, seems to increase bone mass and BMD, and improve markers of bone turnover, while it seems to show no reduction in fracture incidence [41].

Arase et al. [42], retrospectively demonstrate, in postmenopausal women with CLD and osteoporosis that cyclic etidronate therapy within 3 months after osteoporosis diagnosis significantly reduces the appearance of bone fracture $(4.9 \%$ in etidronate group and $13.8 \%$ in control notreated group, not given any drugs for osteoporosis after the diagnosis). No complications from esophageal daily administration of alendronate were observed, although other studies, also conducted in patients affected with PBC, suggest that alendronate is better tolerated when administered once a week, rather than daily [43]. Preliminary results comparing alendronate $70 \mathrm{mg}$ weekly versus ibandronate $150 \mathrm{mg}$ monthly in PBC patients with osteoporosis or low bone mass and fragility fractures show that both drugs have similar effects on BMD, but the adherence to treatment is superior for monthly ibandronate, without adverse effects on liver tests [44]. Contrariwise, Floreani et al. conducted a prospective study on 100 post-menopausal women, affected with $\mathrm{PBC}$, treated with calcium (1,000 mg/day), vitamin D3 (880 IU/day) and i.m. disodium clodronate (100 mg, every 10 days) for 4 years. The BMD was assessed by DEXA at the baseline and every 2 years for up to 4 years.

The treatment did not significantly improve osteoporosis or osteopenia, but prevents the natural bone loss in these patients [45]. In patients affected with non-biliary cirrhosis, bisphosphonates seem, overall, well tolerated in weekly administration, although they must be used with care in patients with a recent history of bleeding or with esophageal sclerotherapy, but these studies were carried out on too small a series to make judgements about their effectiveness in this different context [46]. Some small studies in patients undergoing liver transplant have been conducted to reduce the incidence of fractures after surgery, using

Fig. 2 Bisphosphonates mechanism of action

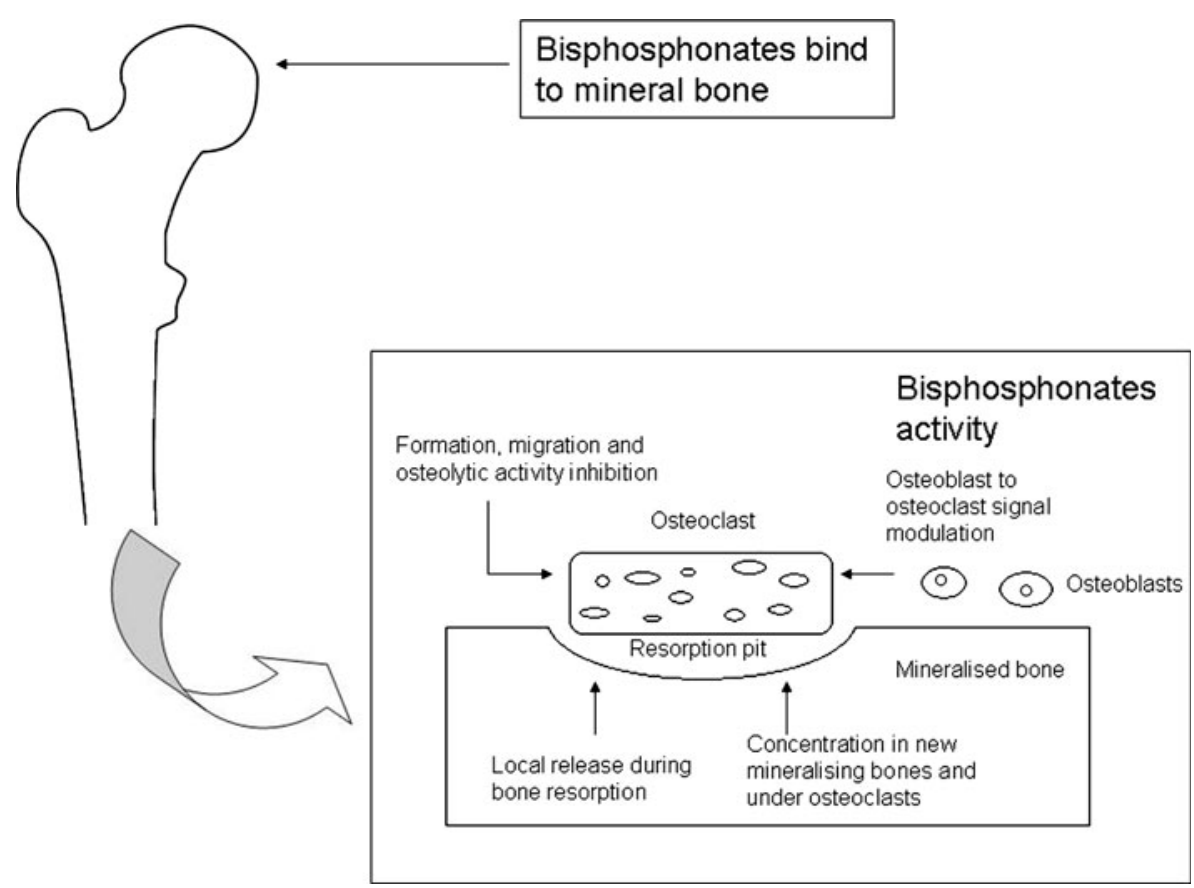


bisphosphonates. Most of them used intravenous pamidronate, others alendronate (weekly administered) and zoledronic acid, obtaining variable results, from a fracture reduction to a BMD rising without change in the incidence of fractures, to neither fracture reduction nor BMD rising. Failure in protective effect detection of these drugs against fracture, found in some of these studies, may reflect, however, only the size of the evaluated sample, and an inappropriate design to detect the capacity of reducing the risk of new fractures, whereas an overall decrease of fracture rate has been observed in post-transplant patients in the last 20 years [5]. In this context, it is important to take care of a particular side effect of bisphosphonate therapy, which is osteonecrosis of the jaw. Most reported cases involve oncologic patients treated with intravenous pamidronate or zoledronic acid, but it has been reported even in patients without cancer in oral treatment. About 2/3 of cases occur after tooth extraction. At date, there is not any knowledge about relevance and prevalence of this adverse reaction in patients with CLD [47].

\section{Other treatments}

\section{Hormone replacement therapy}

Hormone replacement therapy (HRT) is actually a secondline choice therapy for osteoporosis treatment, due to evidence of an increased risk of thromboembolic and gynecologic malignant neoplasm. However, it may be theoretically administered, especially in pre-menopausal women with CLD and documented hypogonadism, or in early menopause women (before 45 years) affected with PBC or autoimmune cirrhosis, or, finally, in postmenopausal women after liver transplantation, usually given by the transdermal route, to increase BMD [48].

\section{Raloxifene}

Raloxifene is an estrogen receptor modulator, which has also been shown to reduce the frequency of vertebral fractures in women with postmenopausal osteoporosis. Contrariwise, it does not seem to affect the frequency of femoral fractures. Currently, there are few data on its safety and efficacy in osteoporotic patients with CLD [49].

\section{Strontium ranelate}

In patients with postmenopausal osteoporosis, strontium ranelate can be successfully used to reduce vertebral and non-vertebral fracture. Its mechanism of action is not well known, and, to date, there are no studies on its efficacy and safety in patients with CLD, especially in those intolerant to bisphosphonates ones [50].

\section{Recombinant PTH}

Recombinant PTH has been used for secondary prevention of fragility fractures in women with postmenopausal osteoporosis, intolerant to bisphosphonate. It works by increasing bone synthesis. To date, there are no studies on its safety and efficacy in patients with CLD [51].

\section{Conclusions and prospectives}

Osteoporosis is the most important form of liver osteodystrophy, especially in the end-stages and in cases with chronic cholestasis, hereditary hemochromatosis and alcohol abuse, often not clinically evident, and, therefore, underdiagnosed. Despite a decline of the osteoporosis incidence in the last 20 years in patients with end-stage CLD, a large number of patients waiting for liver transplant are affected with osteoporosis, and many of them have already had fractures. It is important to identify these individuals, because pre-transplant fractures increase the risk of post-transplant ones, and because fractures, whenever they occur, are associated with great morbidity, even in non-transplanted cirrhotic patients. The pathogenesis of osteoporosis is mainly characterized by low bone formation, particularly related to the effect of retained substances of cholestasis, such as bilirubin and bile acids, or to the iron and alcohol on osteoblastic cells. Increased bone resorption has also been described, especially in cholestatic women with advanced disease. Good nutrition, such as suppression of risk factors, and the administration of supplements of calcium and vitamin $\mathrm{D}$, is strongly recommended for the prevention and treatment of osteoporosis. Evidence of beneficial effects of pharmacological treatment in these particular groups of patients is still, mainly, based on studies conducted in women with postmenopausal osteoporosis, being few in number and with small numbers of subjects in studies on patients with CLD. In particular, bisphosphonates, especially weekly alendronate and monthly ibandronate, appear to improve BMD, and to be well tolerated, but studies in this regard are too small to show a real beneficial effect, even on fracture frequency. The efficacy of bisphosphonates in patients after liver transplantation remains to be confirmed, although appealing effects of pamidronate, alendronate and zoledronic acid have been reported. The outcome in reducing the incidence of fractures has not been adequately demonstrated essentially because of the low number of patients included in the therapeutic trials.

In conclusion, in all CLD patients, especially those with severe cholestasis, and in the presence of a normal BMD, basic preventive measures must be considered (i.e., avoiding excess alcohol intake, smoking cessation, regular 
weight-hearing exercise, and adequate calcium/vitamin D intake, by diet or pharmacological replacement). In this context, physicians must also consider hypogonadism correction, and to repeat DEXA in 2-3 years. If on long-term corticosteroids treatment, minimize the dose, and repeat DEXA in 1 year. If osteopenic, basic preventive measures must be applied plus repeat DEXA in 2-3 years. If on longterm corticosteroid therapy, consider bisphosphonates and repeat DEXA in 1 year. Finally, if CLD patient is osteoporotic, basic preventive measures must be applied, plus screening for other low BMD causes (i.e., laboratory screen: complete blood count, serum creatinine, serum calcium, serum phosphate, alkaline phosphatase, protein electrophoresis, 25-OHD, PTH, thyroid function tests, oestradiol, FSH, LH, and testosterone [in men]), bisphosphonate therapy and repeat DEXA in 1 year. 25

\section{Box}

Box 1: Among patients with chronic liver disease, who should undergo bone mineral density measurement?

- Cholestatic (primary biliary cirrhosis/primary sclerosing cholangitis)

- Hereditary hemochromatosis

- Selected viral (postmenopausal $\mathrm{HCV}+$ patients)

- Before IFN-based therapy, if risk factors are present

- Alcohol

- Liver transplantation

- Before steroids and/or cyclosporin A

Box 2: When should bone mineral density be followed after initial diagnosis

- If abnormal

- If cholestatic

- If on osteopenia inducing drugs

- Every 2-3 years, or every year in high-risk patients (i.e., cholestatic ones)

Box 3: Use of bisphosphonates in patients with chronic liver disease (cirrhosis)

- Size of the problem (prevalence of osteoporosis)

- Need for treatment in the light of natural history (life expectancy)

- Safety of bisphosphonates:

- potential for hepatotoxicity

- drugs-related (consider hepatic and renal function)

- unpredictable

- gastric mucosal problems (portal hypertensive gastropathy) and varices

Conflict of interest None.

\section{References}

1. Sanchez AJ, Aranda-Michel J (2006) Liver disease and osteoporosis. Nutr Clin Pract 21:273-278

2. Pappa HM, Bern E, Kamin D, Grand RJ (2008) Vitamin D status in gastrointestinal and liver disease. Curr Opin Gastroenterol 24: 176-183

3. Arteh J, Narra S, Nair S (2010) Prevalence of vitamin D deficiency in chronic liver disease. Dig Dis Sci 55:2624-2628

4. Dontas IA, Yiannakopoulos CK (2007) Risk factors and prevention of osteoporosis-related fractures. J Musculoskelet Neuronal Interact 7:268-272

5. Collier J (2007) Bone disorders in chronic liver disease. Hepatology 46:1271-1278

6. Stellon AJ, Webb A, Compston J, Williams R (1987) Low bone turnover state in primary biliary cirrhosis. Hepatology 7:137-142

7. Handelsman DJ, Strasser S, McDonald JA, Conway AJ, McCaughan GW (1995) Hypothalamic-pituitary-testicular function in end-stage non-alcoholic liver disease before and after liver transplant. Clin Endocrinol 43:331-337

8. Bell H, Raknerud N, Falch JA, Haug E (1995) Inappropriately low levels of gonadotrophins in amenorrhoeic women with alcoholic and non alcoholic cirrhosis. Eur J Endocrinol 132:444-449

9. Williams FM, Spector TD (2007) The genetics of osteoporosis. Acta Rheumatol Port 32:231-240

10. Moschen AR, Kaser A, Stadlmann S, Millonig G, Kaser S, Mühllechner P, Habior A, Graziadei I, Vogel W, Tilg H (2005) The RANKL/OPG system and bone mineral density in patients with chronic liver disease. J Hepatol 43:973-983

11. Gallego-Rojo FJ, Gonzalez-Calvin JL, Muñoz-Torres M, Mundi JL, Fernandez-Perez R, Rodrigo-Moreno D (1998) Bone mineral density, serum insulin-like growth factor I, and bone turnover markers in viral cirrhosis. Hepatology 28:695-699

12. Cockayne S, Adamson J, Lanham-New S, Shearer MJ, Gilbody S, Torgerson DJ (2006) Vitamin K and the prevention of fractures: systematic review and meta-analysis of randomized controlled trials. Arch Intern Med 166:1256-1261

13. Smith DL, Shire NJ, Watts NB, Schmitter T, Szabo G, Zucker SD (2006) Hyperbilirubinemia is not a major contributing factor to altered bone mineral density in patients with chronic liver disease. J Clin Densitom 9:105-113

14. Ruiz-Gaspa S, Martinez-Ferrer A, Enjuanes A, Peris P, Martinez de Osaba MJ, Alvarez L, Monegal A, Combalia A, Guanabens N, Pares A (2010) High bilirubin reduces cell survival and differentiation of primary human osteoblasts. Is this effect applicable to the serum of chronic cholestatic patients? J Hepatol 52:s214-s215

15. Guañabens N, Cerdá D, Monegal A, Pons F, Caballería L, Peris P, Parés A (2010) Low bone mass and severity of cholestasis affect fracture risk in patients with primary biliary cirrhosis. Gastroenterology 138:2348-2356

16. Menon KV, Angulo P, Weston S, Dickson ER, Lindor KD (2001) Bone disease in primary biliary cirrhosis: independent indicators and rate of progression. J Hepatol 35:316-323

17. Mounach A, Ouzzif Z, Wariaghli G, Achemlal L, Benbaghdadi I, Aouragh A, Bezza A, El Maghraoui A (2008) Primary biliary cirrhosis and osteoporosis: a case-control study. J Bone Miner Metab 26:379-384

18. Solaymani-Dodaran M, Card TR, Aithal GP, West J (2006) Fracture risk in people with primary biliary cirrhosis: a population-based cohort study. Gastroenterology 131:1752-1757

19. Benetti A, Crosignani A, Varenna M, Giussani CS, Allocca M, Zuin M, Podda M, Battezzati PM (2008) Primary biliary cirrhosis is not an additional risk factor for bone loss in women receiving regular calcium and vitamin D supplementation: a controlled longitudinal study. J Clin Gastroenterol 42:306-311 
20. Guañabens N, Parés A, Ros I, Caballería L, Pons F, Vidal S, Monegal A, Peris P, Rodés J (2005) Severity of cholestasis and advanced histological stage but not menopausal status are the major risk factors for osteoporosis in primary biliary cirrhosis. J Hepatol 42:573-577

21. Angulo P, Therneau TM, Jorgensen A, DeSotel CK, Egan KS, Dickson ER, Hay JE, Lindor KD (1998) Bone disease in patients with primary sclerosing cholangitis: prevalence, severity and prediction of progression. J Hepatol 29:729-735

22. Angulo P, Grandison GA, Fong DG, Keach JC, Lindor KD, Bjornsson E, Koch A (2011) Bone disease in patients with primary sclerosing cholangitis. Gastroenterology 140:180-188

23. Guichelaar MM, Malinchoc M, Sibonga J, Clarke BL, Hay JE (2002) Bone metabolism in advanced cholestatic liver disease: analysis by bone histomorphometry. Hepatology 36:895-903

24. Valenti L, Varenna M, Fracanzani AL, Rossi V, Fargion S, Sinigaglia L (2009) Association between iron overload and osteoporosis in patients with hereditary hemochromatosis. Osteoporos Int 20:549-555

25. Loria I, Albanese C, Giusto M, Galtieri PA, Giannelli V, Lucidi C, Di Menna S, Pirazzi C, Corradini SG, Mennini G, Rossi M, Berloco P, Merli M (2010) Bone disorders in patients with chronic liver disease awaiting liver transplantation. Transplant Proc 42:1191-1193

26. Yenice N, Gümrah M, Mehtap O, Kozan A, Türkmen S (2006) Assessment of bone metabolism and mineral density in chronic viral hepatitis. Turk J Gastroenterol 17:260-266

27. Schiefke I, Fach A, Wiedmann M, Aretin AV, Schenker E, Borte G, Wiese M, Moessner J (2005) Reduced bone mineral density and altered bone turnover markers in patients with non-cirrhotic chronic hepatitis B or C infection. World J Gastroenterol 11: 1843-1847

28. Solís-Herruzo JA, Castellano G, Fernández I, Muñoz R, Hawkins F (2000) Decreased bone mineral density after therapy with alpha interferon in combination with ribavirin for chronic hepatitis $\mathrm{C}$. J Hepatol 33:812-817

29. Hofmann WP, Kronenberger B, Bojunga J, Stamm B, Herrmann E, Bücker A, Mihm U, von Wagner M, Zeuzem S, Sarrazin C (2008) Prospective study of bone mineral density and metabolism in patients with chronic hepatitis $\mathrm{C}$ during pegylated interferon alpha and ribavirin therapy. J Viral Hepat 15:790-796

30. Diamond T, Stiel D, Lunzer M, Wilkinson M, Posen S (1989) Ethanol reduces bone formation and may cause osteoporosis. Am J Med 86:282-288

31. Peris P, Guañabens N, Parés A, Pons F, del Rio L, Monegal A, Surís X, Caballería J, Rodés J, Muñoz-Gómez J (1995) Vertebral fractures and osteopenia in chronic alcoholic patients. Calcif Tissue Int 57:111-114

32. Meys E, Fontanges E, Fourcade N, Thomasson A, Pouyet M, Del-mas PD (1994) Bone loss after orthotopic liver transplant. Am J Med 97:445-450

33. Guichelaar MM, Schmoll J, Malinchoc M, Hay JE (2007) Fractures and avascular necrosis before and after orthotopic liver transplantation: long-term follow-up and predictive factors. Hepatology 46:1198-1207

34. Guichelaar MM, Kendall R, Malinchoc M, Hay JE (2006) Bone mineral density before and after OLT: long-term follow-up and predictive factors. Liver Transpl 12:1390-1402

35. Encke J, Uhl W, Stremmel W, Sauer P (2004) Immunosuppression and modulation in liver transplant. Nephrol Dial Transplant 19(Suppl 4):iv22-iv25
36. Leidig-Bruckner G, Hosch S, Dodidou P, Ritschel D, Conradt C, Klose C, Otto G, Lange R, Theilmann L, Zimmerman R, Pritsch M, Ziegler R (2001) Frequency and predictors of osteoporotic fractures after cardiac or liver transplant: a follow-up study. Lancet 357:342-347

37. Collier JD, Ninkovic M, Compston JE (2002) Guidelines on the management of osteoporosis associated with chronic liver disease. Gut 50(Suppl 1):i1-i9

38. Leslie WD, Bernstein CN, American Gastroenterological Association Clinical Practice Committee (2003) AGA technical review on osteoporosis in hepatic disorders. Gastroenterology 125: 941-966

39. Grey A (2007) Emerging pharmacologic therapies for osteoporosis. Expert Opin Emerg Drugs 12:493-508

40. Crawford BA, Labio ED, Strasser SI, McCaughan GW (2006) Vitamin D replacement for cirrhosis related bone disease. Nat Clin Pract Gastroenterol Hepatol 3:689-699

41. Guañabens N, Parés A, Ros I, Alvarez L, Pons F, Caballería L, Monegal A, Martínez de Osaba MJ, Roca M, Peris P, Rodés J (2003) Alendronate is more effective than etidronate for increasing bone mass in osteopenic patients with primary biliary cirrhosis. Am J Gastroenterol 98:2268-2274

42. Arase Y, Suzuki F, Suzuki Y, Akuta N, Kobayashi M, Kawamura Y, Yatsuji H, Sezaki H, Hosaka T, Ikeda K, Kumada H (2008) Prolonged-efficacy of bisphosphonate in postmenopausal women with osteoporosis and chronic liver disease. J Med Virol 80: 1302-1307

43. Zein CO, Jorgensen RA, Clarke B, Wenger DE, Keach JC, Angulo P, Lindor KD (2005) Alendronate improves bone mineral density in primary biliary cirrhosis: a randomized placebo-controlled trial. Hepatology 42:762-771

44. Guanabens N, Cerda D, Monegal A, Muxi A, Caballeria L, Peris P (2010) Monthly ibandronate vs. weekly alendronate in the treatment of osteoporosis associated with primary biliary cirrhosis: similar efficacy but different adherence. J Hepatol 52:S79

45. Floreani A, Carderi I, Ferrara F, Rizzotto ER, Luisetto G, Canozzi V, Baldo V (2007) A 4 year treatment with clodronate plus calcium and vitamin D supplements does not improve bone mass in primary biliary cirrhosis. Dig Liver Dis 39:544-548

46. Owens G, Jackson R, Lewiecki EM (2007) An integrated approach: bisphosphonate management for the treatment of osteoporosis. Am J Manag Care 13(Suppl 11):S290-S308

47. Pazianas M, Miller P, Blumentals WA, Bernal M, Kothawala P (2007) A review of the literature on osteonecrosis of the jaw in patients with osteoporosis treated with oral bisphosphonates: prevalence, risk factors, and clinical characteristics. Clin Ther 29:1548-1558

48. Boone RH, Cheung AM, Girlan LM, Heathcote EJ (2006) Osteoporosis in primary biliary cirrhosis: a randomized trial of the efficacy and feasibility of estrogen/progestin. Dig Dis Sci 51: $1103-1112$

49. Levy C, Harnois DM, Angulo P, Jorgensen R, Lindor KD (2005) Raloxifene improves bone mass in osteopenic women with primary biliary cirrhosis: results of a pilot study. Liver Int 25: $117-121$

50. Blake GM, Fogelman I (2006) Strontium ranelate: a novel treatment for postmenopausal osteoporosis: a review of safety and efficacy. Clin Interv Aging 1:367-375

51. Girotra M, Rubin MR, Bilezikian JP (2006) The use of parathyroid hormone in the treatment of osteoporosis. Rev Endocr Metab Disord 7:113-121 